\title{
MutRank: An R Shiny web-application for exploratory targeted mutual rank-based coexpression analyses integrated with user-provided supporting information
}

\author{
Elly Poretsky ${ }^{1}$, Alisa Huffaker ${ }^{\text {Corresp. } 1}$ \\ ${ }^{1}$ Division of Biology, University of California, San Diego, La Jolla, CA, United States \\ Corresponding Author: Alisa Huffaker \\ Email address: ahuffaker@ucsd.edu
}

The rapid assignment of genotypes to phenotypes has been a historically challenging process. The discovery of genes encoding biosynthetic pathway enzymes for defined plant specialized metabolites has been informed and accelerated by the detection of gene clusters. Unfortunately, biosynthetic pathway genes are commonly dispersed across chromosomes or reside in genes clusters that provide little predictive value. More reliably, transcript abundance of genes underlying biochemical pathways for plant specialized metabolites display significant coregulation. By rapidly identifying highly coexpressed transcripts, it is possible to efficiently narrow candidate genes encoding pathway enzymes and more easily predict both functions and functional associations. Mutual Rank-based coexpression analyses in plants accurately demonstrate functional associations for many specialized metabolic pathways; however, despite the clear predictive value of Mutual Rank analyses, the application is uncommonly used to drive new pathway discoveries. Moreover, many coexpression databases aid in the prediction of both functional associations and gene functions, but lack customizability for refined hypothesis testing. To facilitate and speed flexible Mutual Rank-based hypothesis testing, we developed MutRank, an R Shiny web-application for coexpression analyses. MutRank provides an intuitive graphical user interface with multiple customizable features that integrates userprovided data and supporting information suitable for personal computers. Tabular and graphical outputs facilitate the rapid analyses of both unbiased and user-defined coexpression results that accelerate gene function predictions. We highlight the recent utility of Mutual Rank analyses for functional predictions and discoveries in defining two maize terpenoid antibiotic pathways. Beyond applications in biosynthetic pathway discovery, MutRank provides a simple, customizable and user-friendly interface to enable coexpression analyses relating to a breadth of plant biology inquiries. Data and code are available at GitHub: https://github.com/eporetsky/MutRank. 
1 MutRank: An R Shiny web-application for exploratory targeted Mutual Rank-based

2 coexpression analyses integrated with user-provided supporting information

3

4

5 Elly Poretsky ${ }^{1}$ and Alisa Huffaker ${ }^{1}$

6

$7 \quad{ }^{1}$ Section of Cell and Developmental Biology, University of California at San Diego, La Jolla, CA

892093

9

10 Corresponding author:

11 Alisa Huffaker

12 Email address: ahuffaker@ucsd.edu

13

14 


\section{Abstract}

16 The rapid assignment of genotypes to phenotypes has been a historically challenging process.

17 The discovery of genes encoding biosynthetic pathway enzymes for defined plant specialized

18 metabolites has been informed and accelerated by the detection of gene clusters. Unfortunately,

19 biosynthetic pathway genes are commonly dispersed across chromosomes or reside in genes

20 clusters that provide little predictive value. More reliably, transcript abundance of genes

21 underlying biochemical pathways for plant specialized metabolites display significant

22 coregulation. By rapidly identifying highly coexpressed transcripts, it is possible to efficiently

23 narrow candidate genes encoding pathway enzymes and more easily predict both functions and

24 functional associations. Mutual Rank-based coexpression analyses in plants accurately

25 demonstrate functional associations for many specialized metabolic pathways; however, despite

26 the clear predictive value of Mutual Rank analyses, the application is uncommonly used to drive

27 new pathway discoveries. Moreover, many coexpression databases aid in the prediction of both

28 functional associations and gene functions, but lack customizability for refined hypothesis

29 testing. To facilitate and speed flexible Mutual Rank-based hypothesis testing, we developed

30 MutRank, an R Shiny web-application for coexpression analyses. MutRank provides an intuitive

31 graphical user interface with multiple customizable features that integrates user-provided data

32 and supporting information suitable for personal computers. Tabular and graphical outputs

33 facilitate the rapid analyses of both unbiased and user-defined coexpression results that

34 accelerate gene function predictions. We highlight the recent utility of Mutual Rank analyses for

35 functional predictions and discoveries in defining two maize terpenoid antibiotic pathways.

36 Beyond applications in biosynthetic pathway discovery, MutRank provides a simple,

37 customizable and user-friendly interface to enable coexpression analyses relating to a breadth of 
38 plant biology inquiries. Data and code are available at GitHub:

39 https://github.com/eporetsky/MutRank.

40

41 Introduction

42 Visually-apparent biological complexity is greatly exceeded by the extreme diversity of

43 specialized metabolites made by organisms for the mediation of essential biotic and abiotic

44 interactions (Dixon, 2001; Gershenzon \& Dudareva, 2007; Pichersky \& Lewinsohn, 2011). In

45 plants, the ability to identify and control the production of specialized metabolites has significant

46 implications for human health and agriculture; however, efficient tools aiding in biosynthetic

47 pathway discovery remain limited (Dixon, 2001; Moghe \& Kruse, 2018). Clustering of plant

48 specialized metabolism genes has historically been a useful, but not the sole, indicator of

49 functional associations, and has accelerated the discovery of multiple specialized metabolite

50 biosynthetic pathways (Frey, 1997; Osbourn, 2010; Boutanaev et al., 2015). For the discovery of

51 non-clustered metabolic pathway genes, coexpression analyses have emerged as a powerful

52 predictive tool. Genes in specialized metabolic pathways are often highly coregulated based on

53 developmental, spatial, environmental and complex regulatory controls (Schmelz et al., 2014;

54 Lacchini \& Goossens, 2020). Genes that work together in functional specialized metabolic

55 pathways are likely to require transcriptional coregulation and thus resulting patterns used to

56 predict both functional associations and putative gene functions (Chae et al., 2014; Wisecaver et

57 al., 2017). With increasingly affordable and accessible next generation sequencing technologies,

58 new public and private custom large-scale transcriptomic datasets are routinely generated (Zhou

59 et al., 2020). Studies in plants often generate hundreds and even thousands of transcriptomic

60 samples from different genotypes, developmental stages, tissues and physiological conditions to 
61 understand traits of agronomic significance (Sekhon et al., 2011; Stelpflug et al., 2016; Kremling

62 et al., 2018; Machado et al., 2020). Moreover, genomes and transcriptomes from thousands of

63 plant species are expected to speed large-scale gene expression experiments in poorly understood

64 models (Twyford, 2018; One Thousand Plant Transcriptomes Initiative, 2019). Public and lab-

65 specific transcriptomic resources are far from static, instead they are continuously expanding and

66 dynamic resources that require flexible tools for rapid and effective analyses.

67

68 Many databases and webtools, such as PLEXdb (Dash et al., 2012), Genevestigator (Hruz et al., 69 2008), PLANEX (Yim et al., 2013), CORNET (De Bodt et al., 2010, 2012), ATTED-II

70 (Obayashi et al., 2018), COXPRESdb (Obayashi et al., 2012), RiceFREND (Sato et al., 2013),

71 ePlant (Waese et al., 2017) and STRING (Szklarczyk et al., 2019) have been developed to

72 facilitate gene coexpression analyses. Coexpression analyses in studies and databases often use

73 the Pearson's Correlation Coefficient (PCC) as a measure of coexpression. Mutual Rank (MR),

74 the geometric mean of the ranked PCCs between a pair of genes, has been further proposed as an

75 alternative measure of coexpression to PCC (Obayashi \& Kinoshita, 2009). MR-based

76 coexpression analyses provide better indication of functional associations and are more robust to

77 inconsistencies caused by different microarray data processing methods compared to PCC-based

78 coexpression analyses (Obayashi \& Kinoshita, 2009). Collective findings have driven some

79 coexpression databases to use MR as the primary measure of coexpression (Obayashi et al.,

80 2012, 2018; Sato et al., 2013). When the MR- and PCC-based coexpression databases of multiple

81 plant species from ATTED-II (Obayashi et al., 2018) were converted into coexpression networks

82 and compared, the MR-based coexpression networks were more comparable than PCC-based

83 coexpression networks across species, suggesting that MR-based coexpression networks 
84 accurately represent functional associations (Wisecaver et al., 2017). MR-based coexpression

85 networks enabled the accurate prediction of clusters enriched for enzymes associated with

86 validated plant specialized metabolic pathways (Wisecaver et al., 2017). Wisecaver et al.

87 (Wisecaver et al., 2017) further demonstrate that MR analyses of transcripts are an improved and 88 powerful tool for the functional prediction of unclustered biosynthetic pathway genes to serve as 89 a springboard for hypothesis testing and validation.

90

91 While coexpression databases are useful, few enable flexible hypothesis testing and tool-based

92 simplicity that integrates user-provided data and information. Data integration with coexpression

93 results facilitates the meaningful interpretation of predicted functional associations and

94 assignment of putative gene functions. For example, if a cytochrome P450 monoxygenase (CYP)

95 is hypothesized to perform an oxidation step in a specific biosynthetic pathway, a user might ask

96 "which of all possible $C Y P$ transcripts is most highly coexpressed with an established pathway

97 gene?". More simply stated, any number of user-defined questions of targeted interest can be

98 precisely examined. For any co-regulated process studied, the identification of 2-3 top candidates

99 from a large gene family can greatly narrow efforts required for defined hypothesis testing and

100 iterative re-testing. Towards this goal, we developed an R Shiny web-application, termed

101 MutRank, to facilitate user control over both targeted and non-targeted MR-based coexpression

102 analyses for rapid hypothesis testing. Using the R Shiny framework, we designed a flexible

103 coexpression analysis platform that combines R packages to easily analyze and integrate user-

104 provided expression data and information. Shiny web-applications are also advantageous for

105 generating highly customizable and user-friendly interfaces that can run on most personal

106 computers. In addition to identifying highly coexpressed genes in any user-provided dataset, 
107 MutRank automatically integrates supporting information such as gene annotations, differential108 expression data, predicted protein domains and assigned Gene Ontology terms to provide useful 109 tabular and graphical outputs as foundation for empirical hypothesis testing. Confirmed through

110 diverse approaches, targeted and untargeted MR-based coexpression tools were recently

111 leveraged to narrow gene candidates and accurately predict enzymes within multiple maize

112 antibiotic biosynthetic pathways (Ding et al., 2019, 2020). The goal of MutRank is to provide

113 simple, customizable and readily accessible tools to speed research progress by using exploratory 114 targeted coexpression analyses to predict gene functions and functional associations.

115

116

117

118

119

120

121

122

123

124

125

126

127

128

129

\section{Methods}

Software packages and example supporting information used - MutRank was developed as a web application using the Shiny R package (1.4.0.2) (Chang et al., 2020) that creates the user interface and manages navigation across the different application components (Fig. 1A). It requires $\mathrm{R}$ (3.4.0) and Java (Version 8 Update 261) to be installed by the user, and the following R packages will be automatically installed: shiny (1.4.0.2) (Chang et al., 2020), hypergea (1.3.6) (Boenn, 2018), ontologyIndex (2.5) (Greene, Richardson \& Turro, 2017), reshape2 (1.4.3) (Wickham, 2007), RColorBrewer (1.1-2) (Neuwirth, 2014), data.table (1.12.8) (Dowle \& Srinivasan, 2020), ggplot2 (3.3.0) (Wickham 2016), visNetwork (2.0.9) (Almende B.V., Thieurmel \& Robert, 2019), igraph (1.2.4.2) (Csardi \& Nepusz, 2005) and shinythemes (1.1.2) (Chang, 2018). To explain the features included in MutRank and to understand the required file structures we provide example expression data and supporting information. All the files used for examples are based on the Zea mays inbred B73 (RefGen_v3) genome annotation. The 
130 expression data is from the Expanded Maize Gene Expression Atlas (Stelpflug et al., 2016) (Fig.

131 1A, Supplemental Table 1), gene annotations from the Phytozome database (Goodstein et al.,

132 2012) (Fig. 1A, Supplemental Table 2), and gene symbols from MaizeGDB (Portwood et al.,

133 2019) (Fig. 1A, Supplemental Table 3). Additional supporting information can be selected in the

134 main panel (Fig. 1A). As an example of analyzing a custom dataset, differential expression data

135 was obtained for maize stems 24 hours after treatment with a fungal pathogen, specifically

136 Southern leaf blight (SLB; Cochliobolus heterostrophus) (Ding et al., 2019) (Supplemental

137 Table 4). The predicted Pfam protein domain annotations and GO term assignments are derived

138 from the Phytozome database (Goodstein et al., 2012) (Supplemental Table 5-6). The GO-basic

139 and Plant-GO-Slim ontologies are from the GO Consortium (Ashburner et al., 2000;

140 The Gene Ontology Consortium, 2019). Lists of maize terpene synthases (TPS) (Ding et al.,

141 2020), cytochrome P450s (CYP) (Ding et al., 2019) and Pfam protein domains associated with

142 specialized metabolism (SM) (Wisecaver et al., 2017) were used as categories to assign to

143 coexpressed genes (Supplemental Table 7).

144

145 Calculating Mutual Rank values - MutRank was developed as a user-friendly tool to quickly

146 identify the most highly coexpressed genes based on MR values for any reference gene and

147 expression dataset. One of the limitations of MutRank is that it does not calculate all pair-wise

148 MR values. Unlike coexpression databases that pre-calculate all pair-wise MR values (Obayashi

149 et al., 2012, 2018; Sato et al., 2013), calculating all pair-wise MR values on the resources

150 available on most personal computers is impractical. Instead, MutRank calculates all PCC values

151 between the user-provided reference gene and all other genes to generate a limited list of genes

152 with the highest PCC values (top 200 genes by default, maximum 1000) for which it is feasible 
153 to calculate MR values. This practical trade-off between whole-genome and targeted

154 coexpression analyses allows MutRank to rapidly complete calculations and to run on the

155 resources of most personal computers. In addition to using a single reference gene, MutRank

156 offers two additional methods for user-defined reference gene sets (Fig. 1B-2). The first method

157 calculates the MR values between all genes in the reference gene set. The second method creates

158 a novel compound reference gene from the average, sum, maximum or minimum expression

159 values of the reference gene set. Using compound reference genes is important for capturing pan-

160 genome patterns with key gene family members displaying highly variable expression across the

161 analyzed germplasm (Ding et al., 2020).

162

163 Integrating user-provided supporting information - As an exploratory targeted coexpression

164 analysis tool, MutRank integrates user-provided supporting information with the identified list of

165 coexpressed genes (Fig. 1B). Users can provide gene annotations and symbols as easy-to-read

166 information connected to the identified list of coexpressed genes. Additional supporting

167 information in the form of lists of differentially-expressed genes, predicted Pfam domains and

168 assigned Gene Ontology (GO) terms can be integrated with the coexpressed genes. Users can

169 also define custom categories made from lists of genes, Pfam domains or GO terms. The goal of

170 assigning a gene in the MR-based coexpression results as belonging to any of the categories is to

171 have a noticeable indication that the gene is either present in the gene list or is assigned at least

172 one of the Pfam protein domains or GO terms.

173

174 Tabular and graphical outputs for coexpression analyses - The primary output is provided in

175 the form of an MR coexpression table (Fig. 1C). User-provided supporting information can be 
176 automatically integrated into the table in separate columns for each of the coexpressed genes.

177 The results from the MR coexpression table are used as the basis for three additional informative

178 outputs: heatmap, network graph and a GO enrichment table (Fig. 1C). The heatmap, generated

179 using ggplot2 (Wickham 2016), provides an overview of the distribution of MR values among

180 the top coexpressed genes. The R igraph package (Csardi \& Nepusz, 2005) is used to convert the

181 coexpression table into a coexpression network and to annotate the gene vertices with user-

182 provided data. The network graph visualization is produced with visNetwork package (Almende

183 B.V., Thieurmel \& Robert, 2019) which allows the user to explore a dynamic network

184 representation with supporting information. GO term enrichment is calculated using the

185 hypergeometric test based on the GO database and all genes with MR values below a user-

186 provided threshold (default $\mathrm{MR}<100$ ). The P-values are adjusted for false discovery rate $(\mathrm{FDR})$

187 and the results are presented in a separate table.

188

189

Results

190

191 Example workflow 1: Integrating coexpression analyses of genes encoding a specialized

192

metabolic pathway with supporting information. In maize and other important grain crops,

benzoxazinoids (BXs) are a highly-studied class of nitrogen-containing specialized metabolites

194

with critical roles in plant protection against both herbivores and pathogens (Frey, 1997; Meihls

195

et al., 2013; Wouters et al., 2016). Genes underlying early steps in the maize BX biosynthetic

196 pathway, namely $B x 1$ to $B x 8$, are consitutively expressed in seedlings and drive the production of

genes, $B x 1$ to $B x 5$ and $B x 8$, are located together on chromosome 4 and represent the first 
199 biosynthetic gene cluster ever described in plants (Frey, 1997; Dutartre, Hilliou \& Feyereisen, 200 2012). In contrast to largely constitutive production, the late stage BX pathway, namely $B \times 10$ to

$201 B \times 14$ and encoded enzymes, display stress-inducible regulation resulting in the conversion of

202 DIMBOA substrates to 2-(2-hydroxy-4,7-

203 dimethoxy-1,4-benzoxazin-3-one)- $\beta$-D-glucopyranose (HDMBOA-Glc) and 2-(2-hydroxy-4,7,8-

204 trimethoxy-1,4-benzoxazin-3-one)- $\beta$-D-glucopyranose ( $\left.\mathrm{HDM}_{2} \mathrm{BOA}-\mathrm{Glc}\right)$, which upon aglycone

205 liberation (HDMBOA and $\mathrm{HDM}_{2} \mathrm{BOA}$ ) result in highly unstable bioactive molecules (Maresh,

206 Zhang \& Lynn, 2006; Meihls et al., 2013; Wouters et al., 2016). While displaying complex

207 regulation of early- and late-stage $B x$ genes influenced by development and biotic stress

208 (Cambier, Hance \& de Hoffmann, 2000; Wouters et al., 2016), BX1 to BX14 collectively

209 catalyze the production of multiple glucoside conjugates that can ultimately act as aglycone

210 defenses (Frey, 1997; Jonczyk et al., 2008; Meihls et al., 2013; Handrick et al., 2016). The gene

$211 B x 1$ encodes an indole-3-glycerol phosphate lyase that cleaves indole-3-glycerolphosphate into

212 free indole, acting as the first committed step in the pathway (Frey, 1997).

213

214 As an example to demonstrate both the power and remaining challenges of using Mutual Ranks

215 to associate pathway genes to one another, we use the reference gene list method to investigate

216 the coexpression of $B x l$ with other $B x$ pathway genes (Fig. 2A, Supplemental Table 8). Users

217 can select which supporting information to automatically integrate with the MR coexpression

218 table generated (Fig. 2A). The final coexpression table includes columns with the MR values in

219 reference to the first gene in the list (i.e. $B x 1$ ), gene symbols and gene annotations, and excludes

220 the categories and fold-change columns (Fig. 2A). $B x 6$ and $B x 7$ were excluded from the

221 coexpression analysis as they were not included in the expression dataset used for this analysis. 
222 The coexpression results in the table can be visualized as a coexpression heatmap that readily

223 reveals the highly coexpressed gene cluster of $B x 1$ through $B x 5$ and $B x 8$, as well as separate

224 coexpression of $B x 10, B x 11$ and Bx13 with one another (Fig. 2B). Similar association patterns

225 can also be observed using an interactive coexpression network with an $\mathrm{MR}<100$ threshold for

226 drawing edges between vertices (Fig. 2C). Using the validated BX pathway as a simplistic

227 MutRank example, we demonstrate the following: 1) the ease of observing strong co-expression

228 of early $B x$ pathway genes, 2) the partial coexpression of late $B x$ pathway genes, and 3)

229 remaining challenges of bioinformatically-connecting complex pathways that display differential

230 regulation of early and late steps (Fig. 2D) (Meihls et al., 2013; Handrick et al., 2016).

231 Importantly, biosynthetic pathways function within the complex context of a living cell. The

232 value in confirming established coexpression patterns is to first undertand how the user-defined

233 dataset is performing. When compelling, these results then encourage further interrogation to

234 address diverse hypotheses and complex surrounding processes, potentially identifying

235 coexpressed transcription factors, transporters, or detoxification enzymes to investigate (Lacchini

$236 \&$ Goossens, 2020).

237

238 Example workflow 2: Using MutRank to predict enzymes in specialized metabolism. In the

239 first example, we used BX-related defenses which have been studied in maize and other cereals

240 for over 60 years (Virtanen et al., 1955; Smissman, LaPidus \& Beck, 1957). More recently,

241 maize diterpenoid pathways have been implicated in diverse protective roles providing fungal,

242 insect and drought resistance (Schmelz et al., 2011; Vaughan et al., 2015; Christensen et al.,

243 2018; Ding et al., 2019). Biosynthesis of protective ent-kaurane-related diterpenoids in maize,

244 termed kauralexins, are mediated by multi-gene terpene synthase (TPS) and cytochrome P450 
245 (CYP) families. Using MR-based coexpression analyses for discovery purposes (Ding et al., 246 2019) we examined one reference gene termed anther ear 2 (ZmAN2) (Supplemental Table 8), 247 that encodes an ent-copalyl diphosphate synthase (ent-CPS) responsible for the cyclization of 248 geranylgeranyl diphosphate into bicyclic pathway precursor ent-copalyl diphosphate (ent-CPP) 249 (Harris et al., 2005). Derived from 2 different genes encoding ent-CPS, ent-CPP is a key 250 substrate shared by the kauralexin, dolabralexin and gibberellin biosynthetic pathways in maize 251 (Mafu et al., 2018; Ding et al., 2019). Using ZmAN2 as a reference gene, we calculated the non252 targeted MR values between the top 200 coexpressed genes and integrated the supporting 253 information (Fig. 3A). For simplification, we then selected the first 12 coexpressed genes and 254 identified 1 TPS gene (Fig. 3A and 3B: diamond shaped vertex), a type I diterpene synthase: 255 kaurene synthase-like 2 (ZmKSL2) and 2 CYP genes (Fig. 3A and 3B: square shaped vertices), $256 Z m C Y P 71 Z 18$ and kaurene oxidase 2 ( $\mathrm{ZmKO}$ ) that were highly coexpressed (Fig. 3A-C). A 257 GO-term enrichment analysis of the MR-based coexpression results using the GO-basic database 258 revealed an enrichment of terms associated with defense responses and terpene synthesis (Fig. 259 3D). With candidates identified through similar MR-based coexpression relationships to those 260 currently presented (Fig. 3A-E), a recent study of kauralexin biosynthetic enzymes were 261 systematically validated using genome wide association studies, heterologous enzyme co262 expression assays, proteomics and characterization of defined genetic mutants (Ding et al., 263 2019). Two additional genes with defined roles in kauralexin biosynthesis that did not match any 264 of the supporting information categories are the $Z m C Y P 71 Z 16$ that is absent from the currently 265 selected expression dataset and the coexpressed kauralexin reductase2 (ZmKR2) encoding a 5 $\alpha$ 266 steroid reductase that saturates B-series kauralexins (Fig. 3A-C) (Ding et al., 2019). Together the 267 combined use of MR analyses with biochemistry and defined genetic mutants defined roles for 
268

269 and anti-pathogen defense enabling rapid assembly of the entire pathway (Fig. 3E) (Ding et al.,

270 2019). Additional genes identified in the MR-based coexpression analysis encode predicted

271 carrier proteins, pathogenesis-related proteins and kinases that might further contribute to the

272 regulation and transport of diterpenoids (Fig. 3A-C). In summary, straightforward MR analyses

273 via MutRank provide a powerful starting point for defining networks surrounding specialized

274 metabolism.

275

276 Discussion

277 MutRank is a user-friendly and powerful tool for exploratory targeted gene coexpression

278 analyses. MutRank enables the simple calculation of MR values for any reference gene or gene

279 set from user-provided expression data. The Shiny web-application interface is ideal for

280 combining MR-based coexpression analyses with useful R packages that produce informative

281 tabular and graphical outputs. We implemented a number of features that allow users to integrate

282 supporting information with the results of the coexpression analyses to facilitate prediction of

283 putative gene functions. Example workflow 1 surveyed genes in the well-established maize BX

284 biosynthetic pathway. Many of these genes were identified and characterized without the benefit

285 of large-scale transcriptomic data (Frey, 1997). The lack of coexpression connections between

286 early and late stage $B x$ biosynthetic genes (Fig. 2A-D) likely provides a partial explaination for

287 the relatively recent discovery of the terminal steps (Meihls et al., 2013; Handrick et al., 2016).

288

289 Public coexpression databases and tools, such as MutRank, provide intuitive user control over

290 MR-based coexpression analyses to drive predictions and hypothesis testing of genes with

Peer) reviewing PDF | (2020:08:52213:1:0:NEW 25 Sep 2020) 
291 currently unknown functions. Example workflow 2 was given as an example where MR-based

292 coexpression analyses were used to guide recent hypothesis testing, and through a combination

293 of diverse approaches, were demonstrated to correctly predict gene functions in the maize

294 kauralexin antibiotic pathway (Ding et al., 2019). Importantly, we note here that custom use of

295 further expression datasets were used to correctly predict the function of an additional kauralexin

296 biosynthetic genes ( $Z m C Y P 71 Z 16)$ within the pathway using MR-based coexpression analyses

297 (Ding et al., 2019). In Ding et al. 2019 the expression datasets were derived from the National

298 Center for Biotechnology Information Sequence Read Archive project IDs SRP115041 and

299 SRP011480. This esoteric detail speaks to an essentail point. Different MR coexpression patterns

300 can be found in related datasets depending on sample size, plant growth conditions, genotypes

301 used, tissue types, cell types, developemental age, presence or absence of biotic or aboitic stress

302 and countless other factors important to the questions being examined. Given this, aggregate

303 estimations of gene co-expression available on public websites typically fall short in facilitating

304 elucidation of relationships of interest. Rapid progress requires flexible control over the analyses

305 of precise data subsets or of larger aggregated datasets for cross-comparison. MutRank allows

306 for a large number of different datasets to be selected, and quickly analzyed and assessed. Most

307 commonly, the search for meaningful coexpression relationships, whether of biosynthetic genes

308 or for more complex regulatory processes, is a guided and highly iterative discovery process,

309 relying on partial insights from related experimental systems. A common goal is to generate

310 high-quality gene candidates for improved hypothesis testing that ultimately informs more

311 expensive and time-consuming in planta analyses of defined mutants. As a further recent

312 example, MR-based coexpression analyses were leveraged and played a key role in defining and

313 disentangling a challenging 10-gene maize sesquiterpenoid antibiotic pathway partially sharing 
314 kauralexin biosynthetic genes (Ding et al., 2020). Research progress in plant specialized

315 metabolism requires rapid, flexible and easy-to-use tools, through which diverse users of varying

316 expertise levels can quickly compare results generated from public or customized user-provided

317 datasets. We now routinely utilize MutRank as a rapid tool for exploratory targeted coexpression

318 analyses facilitating the prediction of functional associations and putative gene functions. The

319 goal of our current effort was to expand the ease and use of the R Shiny web-application tools to

320 facilitate efforts of any biologists who seek to connect coregulated genes to important

321 phenotypes.

322

323 Conclusion

324 The MutRank R Shiny web application provides an efficient, flexible and simple tool for

325 conducting hypothesis-driven MR-based coexpression analyses. To enable rapid functional

326 discovery, MutRank analyses are integrated with multiple customizable features for narrowing

327 and prioritizing candidate genes and for hypothesis testing in predicted biochemical functions.

328

329 Data Deposition

330 Data and code are available at GitHub: https://github.com/eporetsky/MutRank

331

332 REFERENCES

333 Almende B.V., Thieurmel B, Robert T. 2019. visNetwork: Network Visualization using "vis.js"

$334 \quad$ Library.

335 Ashburner M, Ball CA, Blake JA, Botstein D, Butler H, Cherry JM, Davis AP, Dolinski K, Dwight SS, 
JE, Ringwald M, Rubin GM, Sherlock G. 2000. Gene Ontology: tool for the unification of biology. Nature Genetics 25:25-29. DOI: 10.1038/75556.

339

340

341

342

343

344

345

346

347

348

349

350

351

352

353

354

355

356

357

358

Boenn M. 2018. hypergea: Hypergeometric Tests.

Boutanaev AM, Moses T, Zi J, Nelson DR, Mugford ST, Peters RJ, Osbourn A. 2015. Investigation of terpene diversification across multiple sequenced plant genomes. Proceedings of the National Academy of Sciences 112:E81-E88. DOI: 10.1073/pnas.1419547112.

Cambier V, Hance T, de Hoffmann E. 2000. Variation of DIMBOA and related compounds content in relation to the age and plant organ in maize. Phytochemistry 53:223-229. DOI: 10.1016/S0031-9422(99)00498-7.

Chae L, Kim T, Nilo-Poyanco R, Rhee SY. 2014. Genomic Signatures of Specialized Metabolism in Plants. Science 344:510-513. DOI: 10.1126/science.1252076.

Chang W. 2018. shinythemes: Themes for Shiny.

Chang W, Cheng J, Allaire JJ, Xie Y, McPherson J. 2020. shiny: Web Application Framework for $R$. Christensen SA, Sims J, Vaughan MM, Hunter C, Block A, Willett D, Alborn HT, Huffaker A, Schmelz EA. 2018. Commercial hybrids and mutant genotypes reveal complex protective roles for inducible terpenoid defenses in maize. Journal of Experimental Botany 69:1693-1705. DOI: 10.1093/jxb/erx495.

Csardi G, Nepusz T. 2005. The igraph software package for complex network research. Complex Systems:1695.

Dash S, Van Hemert J, Hong L, Wise RP, Dickerson JA. 2012. PLEXdb: gene expression resources for plants and plant pathogens. Nucleic Acids Research 40:D1194-D1201. DOI: 10.1093/nar/gkr938. 
359 De Bodt S, Carvajal D, Hollunder J, Van den Cruyce J, Movahedi S, Inzé D. 2010. CORNET: A

360 User-Friendly Tool for Data Mining and Integration. Plant Physiology 152:1167-1179.

$361 \quad$ DOI: $10.1104 / p p .109 .147215$.

362 De Bodt S, Hollunder J, Nelissen H, Meulemeester N, Inzé D. 2012. CORNET 2.0: integrating

363 plant coexpression, protein-protein interactions, regulatory interactions, gene

364 associations and functional annotations. New Phytologist 195:707-720. DOI:

365 10.1111/j.1469-8137.2012.04184.x.

366

367

368

369

370

371

372

373

374

375

376

377

378

Ding Y, Murphy KM, Poretsky E, Mafu S, Yang B, Char SN, Christensen SA, Saldivar E, Wu M, Wang Q, Ji L, Schmitz RJ, Kremling KA, Buckler ES, Shen Z, Briggs SP, Bohlmann J, Sher A, Castro-Falcon G, Hughes CC, Huffaker A, Zerbe P, Schmelz EA. 2019. Multiple genes recruited from hormone pathways partition maize diterpenoid defences. Nature Plants 5:1043-1056. DOI: 10.1038/s41477-019-0509-6.

Ding Y, Weckwerth PR, Poretsky E, Murphy KM, Sims J, Saldivar E, Christensen SA, Char SN, Yang B, Tong A, Shen Z, Kremling KA, Buckler ES, Kono T, Nelson DR, Bohlmann J, Bakker MG, Vaughan MM, Khalil AS, Betsiashvili M, Briggs SP, Zerbe P, Schmelz EA, Huffaker A. 2020. Genetic elucidation of complex biochemical traits mediating maize innate immunity. Plant Biology. DOI: 10.1101/2020.03.04.977355.

Dixon RA. 2001. Natural products and plant disease resistance. Nature 411:843-847. DOI: $10.1038 / 35081178$.

Dowle M, Srinivasan A. 2020. data.table: Extension of 'data.frame`. 
379 Dutartre L, Hilliou F, Feyereisen R. 2012. Phylogenomics of the benzoxazinoid biosynthetic

380

381

382

383

384

385

386

387

388

389

390

391

392

393

394

395

396

397

398

399

pathway of Poaceae: gene duplications and origin of the Bx cluster. BMC Evolutionary

Biology 12:64. DOI: 10.1186/1471-2148-12-64.

Frey M. 1997. Analysis of a Chemical Plant Defense Mechanism in Grasses. Science 277:696699. DOI: $10.1126 /$ science.277.5326.696.

The Gene Ontology Consortium. 2019. The Gene Ontology Resource: 20 years and still GOing strong. Nucleic Acids Research 47:D330-D338. DOI: 10.1093/nar/gky1055.

Gershenzon J, Dudareva N. 2007. The function of terpene natural products in the natural world. Nature Chemical Biology 3:408-414. DOI: 10.1038/nchembio.2007.5.

Goodstein DM, Shu S, Howson R, Neupane R, Hayes RD, Fazo J, Mitros T, Dirks W, Hellsten U, Putnam N, Rokhsar DS. 2012. Phytozome: a comparative platform for green plant genomics. Nucleic Acids Research 40:D1178-D1186. DOI: 10.1093/nar/gkr944.

Greene D, Richardson S, Turro E. 2017. ontologyX: a suite of R packages for working with ontological data. Bioinformatics 33:1104-1106. DOI: 10.1093/bioinformatics/btw763.

Handrick V, Robert CAM, Ahern KR, Zhou S, Machado RAR, Maag D, Glauser G, FernandezPenny FE, Chandran JN, Rodgers-Melnick E, Schneider B, Buckler ES, Boland W, Gershenzon J, Jander G, Erb M, Köllner TG. 2016. Biosynthesis of 8-O-methylated benzoxazinoid defense compounds in maize. The Plant Cell 28:1682-1700. DOI: 10.1105/tpc.16.00065.

Harris L, Saparno A, Johnston A, Prisic S, Xu M, Allard S, Kathiresan A, Ouellet T, Peters RJ. 2005. The Maize An2 Gene is Induced by Fusarium Attack and Encodes an ent-Copalyl 
Diphosphate Synthase. Plant Molecular Biology 59:881-894. DOI: 10.1007/s11103-005-

401 1674-8.

402 Hruz T, Laule O, Szabo G, Wessendorp F, Bleuler S, Oertle L, Widmayer P, Gruissem W, 403 Zimmermann P. 2008. Genevestigator V3: A Reference Expression Database for the

404 Meta-Analysis of Transcriptomes. Advances in Bioinformatics 2008:1-5. DOI:

405 $10.1155 / 2008 / 420747$

406

407

408

409

410

411

412

413

414

415

416

417

418

419

420

421
Buckler ES. 2018. Dysregulation of expression correlates with rare-allele burden and fitness loss in maize. Nature 555:520-523. DOI: 10.1038/nature25966.

Lacchini E, Goossens A. 2020. Combinatorial Control of Plant Specialized Metabolism: Mechanisms, Functions, and Consequences. Annual Review of Cell and Developmental Biology 36:1-23. DOI: 10.1146/annurev-cellbio-011620-031429.

Machado FB, Moharana KC, Almeida-Silva F, Gazara RK, Pedrosa-Silva F, Coelho FS, Grativol C, Venancio TM. 2020. Systematic analysis of 1298 RNA-Seq samples and construction of a comprehensive soybean ( Glycine max ) expression atlas. The Plant Journal 103:18941909. DOI: $10.1111 /$ tpj.14850.

Mafu S, Ding Y, Murphy KM, Yaacoobi O, Addison JB, Wang Q, Shen Z, Briggs SP, Bohlmann J, Castro-Falcon G, Hughes CC, Betsiashvili M, Huffaker A, Schmelz EA, Zerbe P. 2018. 

Defenses in Maize. Plant Physiology 176:2677-2690. DOI: 10.1104/pp.17.01351.

424

425

426

427

428

429

430

431

432

433

434

435

436

437

438

439

440

441

442

443

Maresh J, Zhang J, Lynn DG. 2006. The Innate Immunity of Maize and the Dynamic Chemical Strategies Regulating Two-Component Signal Transduction in Agrobacterium tumefaciens. ACS Chemical Biology 1:165-175. DOI: 10.1021/cb600051w.

Meihls LN, Handrick V, Glauser G, Barbier H, Kaur H, Haribal MM, Lipka AE, Gershenzon J, Buckler ES, Erb M, Kollner TG, Jander G. 2013. Natural Variation in Maize Aphid Resistance Is Associated with 2,4-Dihydroxy-7-Methoxy-1,4-Benzoxazin-3-One Glucoside Methyltransferase Activity. The Plant Cell 25:2341-2355. DOI: 10.1105/tpc.113.112409.

Moghe GD, Kruse LH. 2018. The study of plant specialized metabolism: Challenges and prospects in the genomics era. American Journal of Botany 105:959-962. DOI: 10.1002/ajb2.1101.

Neuwirth E. 2014. RColorBrewer: ColorBrewer Palettes.

Obayashi T, Aoki Y, Tadaka S, Kagaya Y, Kinoshita K. 2018. ATTED-II in 2018: A Plant Coexpression Database Based on Investigation of the Statistical Property of the Mutual Rank Index. Plant and Cell Physiology 59:e3(1-7). DOI: 10.1093/pcp/pcx191.

Obayashi T, Kinoshita K. 2009. Rank of Correlation Coefficient as a Comparable Measure for Biological Significance of Gene Coexpression. DNA Research 16:249-260. DOI: 10.1093/dnares/dsp016.

Obayashi T, Okamura Y, Ito S, Tadaka S, Motoike IN, Kinoshita K. 2012. COXPRESdb: a database of comparative gene coexpression networks of eleven species for mammals. Nucleic Acids Research 41:D1014-D1020. DOI: 10.1093/nar/gks1014. 
444 One Thousand Plant Transcriptomes Initiative. 2019. One thousand plant transcriptomes and 445 the phylogenomics of green plants. Nature 574:679-685. DOI: 10.1038/s41586-019-

446 1693-2.

447 Osbourn A. 2010. Gene Clusters for Secondary Metabolic Pathways: An Emerging Theme in 448 Plant Biology: Figure 1. Plant Physiology 154:531-535. DOI: 10.1104/pp.110.161315.

449 Pichersky E, Lewinsohn E. 2011. Convergent Evolution in Plant Specialized Metabolism. Annual 450 Review of Plant Biology 62:549-566. DOI: 10.1146/annurev-arplant-042110-103814.

451

452

453

454

455

456

457

458

459

460

461

462

463

464

465

Portwood JL, Woodhouse MR, Cannon EK, Gardiner JM, Harper LC, Schaeffer ML, Walsh JR, Sen TZ, Cho KT, Schott DA, Braun BL, Dietze M, Dunfee B, Elsik CG, Manchanda N, Coe E, Sachs M, Stinard P, Tolbert J, Zimmerman S, Andorf CM. 2019. MaizeGDB 2018: the maize multi-genome genetics and genomics database. Nucleic Acids Research 47:D1146-D1154. DOI: 10.1093/nar/gky1046.

Sato Y, Namiki N, Takehisa H, Kamatsuki K, Minami H, Ikawa H, Ohyanagi H, Sugimoto K, Itoh J-I, Antonio BA, Nagamura Y. 2013. RiceFREND: a platform for retrieving coexpressed gene networks in rice. Nucleic Acids Research 41:D1214-D1221. DOI: 10.1093/nar/gks1122.

Schmelz EA, Huffaker A, Sims JW, Christensen SA, Lu X, Okada K, Peters RJ. 2014. Biosynthesis, elicitation and roles of monocot terpenoid phytoalexins. The Plant Journal 79:659-678. DOI: 10.1111/tpj.12436.

Schmelz EA, Kaplan F, Huffaker A, Dafoe NJ, Vaughan MM, Ni X, Rocca JR, Alborn HT, Teal PE. 2011. Identity, regulation, and activity of inducible diterpenoid phytoalexins in maize. Proceedings of the National Academy of Sciences 108:5455-5460. DOI: 10.1073/pnas.1014714108. 
466 Sekhon RS, Lin H, Childs KL, Hansey CN, Buell CR, de Leon N, Kaeppler SM. 2011. Genome-wide 467 atlas of transcription during maize development: Maize gene atlas. The Plant Journal 468 66:553-563. DOI: 10.1111/j.1365-313X.2011.04527.x.

469 Smissman E, LaPidus J, Beck S. 1957. Notes - Corn Plant Resistance Factor. The Journal of 470 Organic Chemistry 22:220-220. DOI: 10.1021/jo01353a036.

471 Stelpflug SC, Sekhon RS, Vaillancourt B, Hirsch CN, Buell CR, de Leon N, Kaeppler SM. 2016. An

472

473

474

475

476

477

478

479

480

481

482

483

484

485 Expanded Maize Gene Expression Atlas based on RNA Sequencing and its Use to Explore Root Development. The Plant Genome 9:1-16. DOI: 10.3835/plantgenome2015.04.0025.

Szklarczyk D, Gable AL, Lyon D, Junge A, Wyder S, Huerta-Cepas J, Simonovic M, Doncheva NT, Morris JH, Bork P, Jensen $\amalg$, Mering C von. 2019. STRING v11: protein-protein association networks with increased coverage, supporting functional discovery in genome-wide experimental datasets. Nucleic Acids Research 47:D607-D613. DOI: 10.1093/nar/gky1131.

Twyford AD. 2018. The road to 10,000 plant genomes. Nature Plants 4:312-313. DOI: 10.1038/s41477-018-0165-2.

Vaughan MM, Christensen S, Schmelz EA, Huffaker A, Mcauslane HJ, Alborn HT, Romero M, Allen LH, Teal PEA. 2015. Accumulation of terpenoid phytoalexins in maize roots is associated with drought tolerance: Maize root phytoalexins play a role in drought tolerance. Plant, Cell \& Environment 38:2195-2207. DOI: 10.1111/pce.12482. 
486 Virtanen Al, Hietala PK, Lundén R, Prydz H. 1955. 2(3)-Benzoxazolinone, an Anti-Fusarium 487 Factor in Rye Seedlings. Acta Chemica Scandinavica 9:1543-1544. DOI: 10.3891/acta.chem.scand.09-1543b.

489

490

491

492

493

494

495

496

497

498

499

500

501

502

503

504

505

506

507

Waese J, Fan J, Pasha A, Yu H, Fucile G, Shi R, Cumming M, Kelley LA, Sternberg MJ, Krishnakumar V, Ferlanti E, Miller J, Town C, Stuerzlinger W, Provart NJ. 2017. ePlant: Visualizing and Exploring Multiple Levels of Data for Hypothesis Generation in Plant Biology. The Plant Cell 29:1806-1821. DOI: 10.1105/tpc.17.00073.

Wickham H. 2007. Reshaping Data with the reshape Package. Journal of Statistical Software 21:1-20.

Wickham H. 2016. ggplot2: Elegant Graphics for Data Analysis. Springer-Verlag New York.

Wisecaver JH, Borowsky AT, Tzin V, Jander G, Kliebenstein DJ, Rokas A. 2017. A Global Coexpression Network Approach for Connecting Genes to Specialized Metabolic Pathways in Plants. The Plant Cell 29:944-959. DOI: 10.1105/tpc.17.00009.

Wouters FC, Blanchette B, Gershenzon J, Vassão DG. 2016. Plant defense and herbivore counter-defense: benzoxazinoids and insect herbivores. Phytochemistry Reviews 15:1127-1151. DOI: 10.1007/s11101-016-9481-1.

Yim WC, Yu Y, Song K, Jang CS, Lee B-M. 2013. PLANEX: the plant co-expression database. BMC Plant Biology 13:1-9. DOI: 10.1186/1471-2229-13-83.

Zhou P, Li Z, Magnusson E, Gomez Cano F, Crisp PA, Noshay JM, Grotewold E, Hirsch CN, Briggs SP, Springer NM. 2020. Meta Gene Regulatory Networks in Maize Highlight Functionally Relevant Regulatory Interactions. The Plant Cell 32:1377-1396. DOI: 10.1105/tpc.20.00080. 


\section{Figure 1}

MutRank interface and workflow chart.

(A) MutRank workflow starts at the (1) Data Input tab at the top navigation bar that allows the selection of files to load and access to different sections of MutRank. In the side panel users can select (2) expression data files and (3) gene descriptions and symbol annotations. In the main panel (4) users can select additional supporting data which includes differentialexpression data, custom categories, protein domains, and the Gene Ontology (GO) GO database along GO assignments. (B) With the user-provided expression data and integrated supporting information users can then select a single target reference gene or gene list to produce a (C) Mutual Rank-based coexpression table and to view the coexpression analysis results as a coexpression heatmap, coexpression network and a GO term enrichment table. 


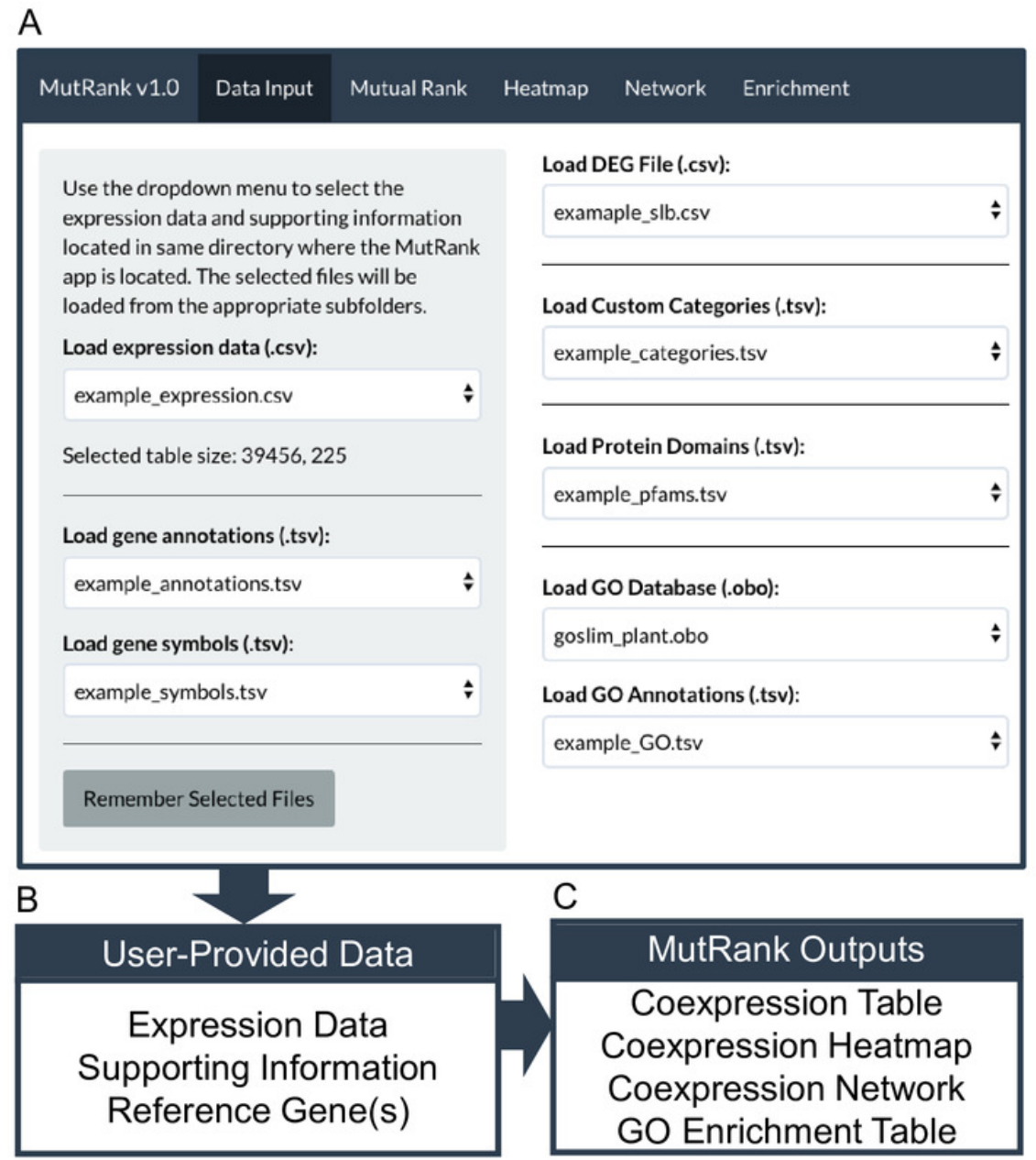

Figure 1. MutRank interface and workflow chart. (A) MutRank workflow starts at the Data Input tab at the top navigation bar that allows the selection of files to load and access to different sections of MutRank. In the side panel users can select expression data files, gene descriptions and symbol annotations. In the main panel users can select additional supporting information which includes differential-expression data, custom categories, protein domains, and the Gene Ontology (GO) database along GO assignments. (B) With the userprovided expression data and integrated supporting information users can then select a single target reference gene or gene list to produce a (C) Mutual Rank-based coexpression table and to view the coexpression analysis results as a coexpression heatmap, coexpression network and a GO term enrichment table. 


\section{Figure 2}

Example workflow 1: Validation of MutRank using a characterized biosynthetic pathway.

(A) In the Mutual Rank (MR) tab we used the reference gene list method (1) with the characterized known enzymes in the Benzoxazinoid (BX) biosynthetic pathway ( $B \times 1$ to $B \times 14$, note: $B \times 6$ and $B \times 7$ are absent from the example expression data) (2) with default output, but excluding custom categories and fold-change values (3) to calculate the MR values and produce the MR coexpression table integrated with supporting information (4). The coexpression analysis results can be presented as a (B) coexpression heatmap and as a (C) coexpression network with an MR $\leq 25$ threshold for drawing edges between vertices: two clusters of coexpressed Bx genes are observed. (D) Summarized diagram of the maize benzoxazinoid biosynthesis pathway. Genes from the reference gene list that were highly coexpressed are designated in red. 


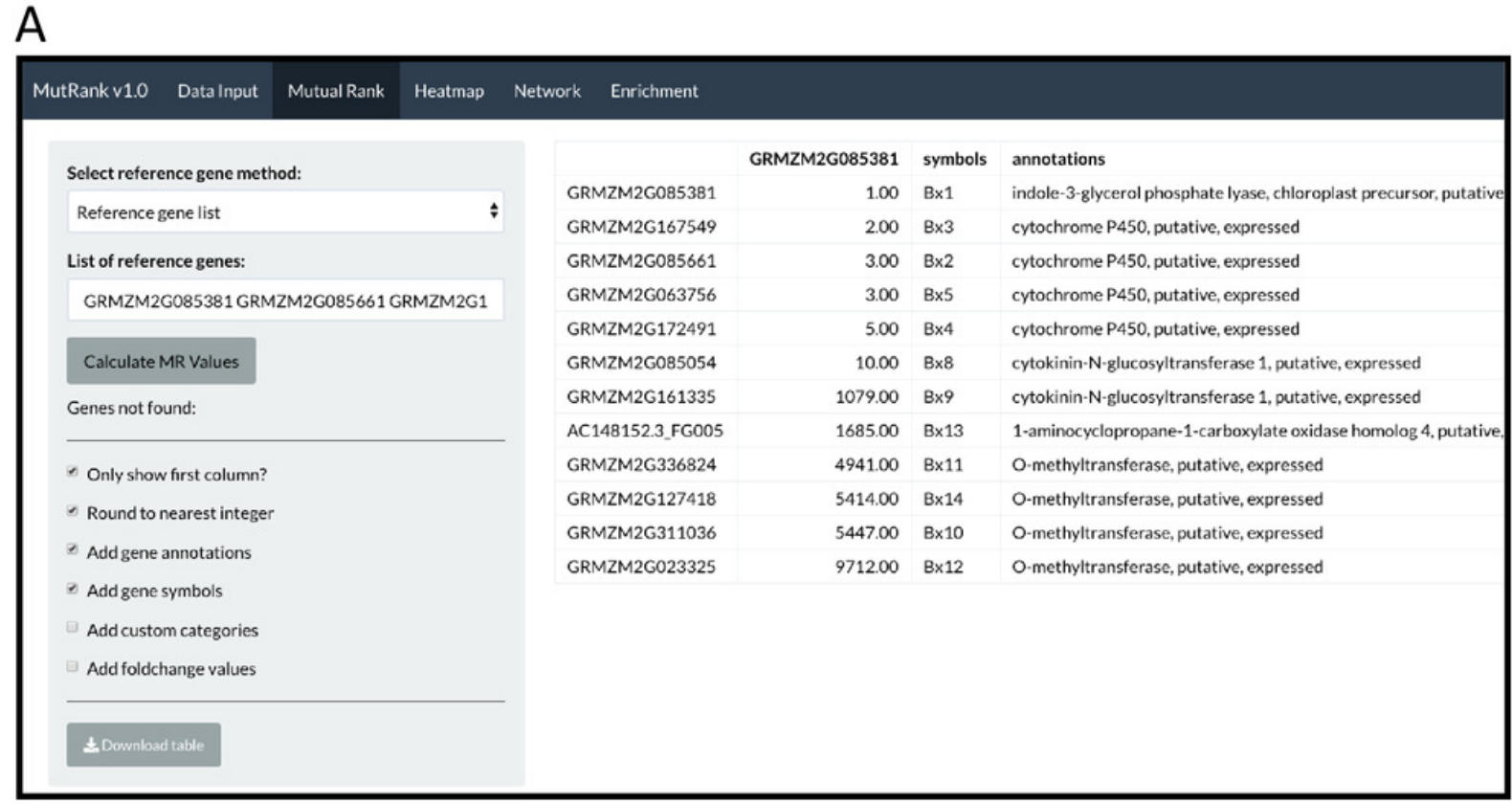

\section{B}

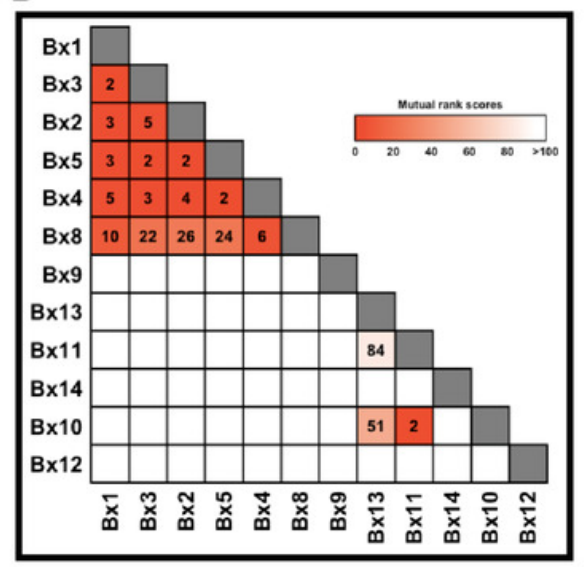

C

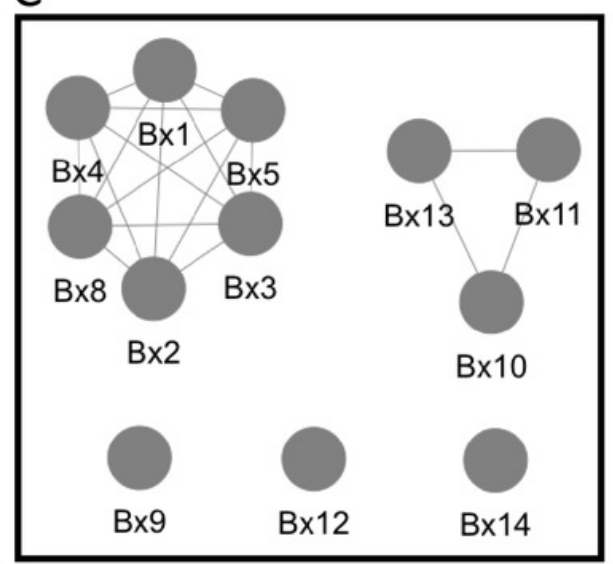

D

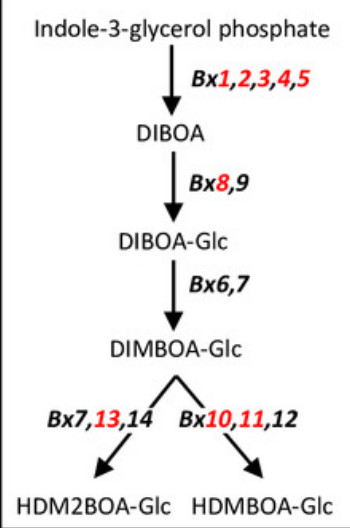

Figure 2. Integrating coexpression analyses of genes encoding a specialized metabolic pathway with supporting information. (A) In the Mutual Rank (MR) tab we used the reference gene list method with the characterized known enzymes in the BX biosynthetic pathways ( $B \times 1$ to $B \times 14$, note: $B \times 6$ and $B \times 7$ are absent from the example expression data) with default output, but excluding custom categories and fold-change values to calculate the MR values and produce the MR coexpression table integrated with supporting information. The coexpression analysis results can be presented as a (B) coexpression heatmap and as a (C) coexpression network with an MR $<100$ threshold for drawing edges between vertices: two clusters of coexpressed Bx genes are observed. (D) Summarized diagram of the maize benzoxazinoid biosynthesis pathway. Genes from the reference gene list that were highly coexpressed are designated in red. 


\section{Figure 3}

Example workflow 2: Using MutRank to predict enzymes in specialized metabolism.

(A) Using the kauralexin biosynthetic gene ANTHER EAR 2 (AN2) as a single reference gene, a Mutual Rank (MR)-based coexpression table was generated for the 200 most highly coexpressed genes (first 12 genes are shown) with the integrated supporting information. Using the first 12 genes in the list we generated a (B) coexpression network figure, with an $M R \leq 10$ threshold for drawing edges between vertices, showing a cluster of coexpressed genes and a (C) coexpression heatmap. $(A, B)$ Genes belonging to a category are denoted with "Y"; the categories used are SM (specialized metabolism), TPS (terpene synthases, diamond shape vertices) and CYP (cytochrome P450s, square shaped vertices). (A,B) Corresponding expression fold change (FC) increase 24 hours after pathogen inoculation. (D) Results of Gene Ontology (GO) GO term enrichment analysis using the GO-basic database are over-represented for biotic stress responses. The analysis included a set of 124 genes with $M R \leq 100$. P-values were calculated with a hypergeometric test using the full GO database and adjusted using the Bonferroni-Holm method. (E) Summarized diagram of the maize kauralexin biosynthetic pathway showing genes highly coexpressed with the reference gene AN2 in red, KAURENE SYNTHASE-LIKE 2 (KSL2), KAURENE OXIDASE 2 (KO2), CYP71Z18 (Z18), and KAURENE REDUCTASE 2 (KR2). 
A

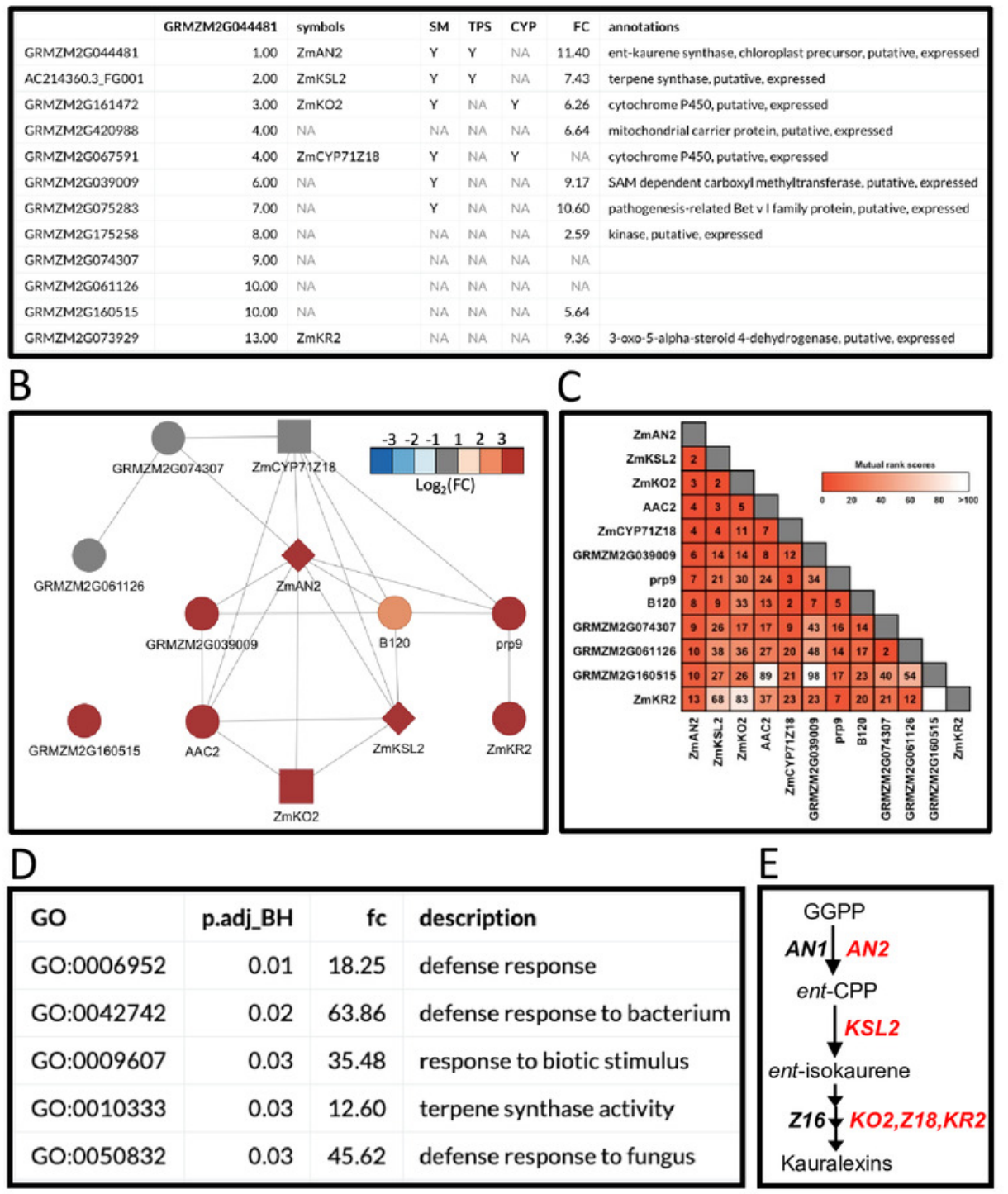

Figure 3. Example workflow 2: Using MutRank to predict enzymes in specialized metabolism. (A) Using AN2 as a single reference gene we generated a Mutual Rank (MR)based coexpression table for the 200 most highly coexpressed genes (first 12 genes are shown) with the integrated supporting information. Using the first 12 genes in the list we generated a (B) coexpression network figure, with an $M R<10$ threshold for drawing edges between vertices, showing a cluster of coexpressed genes and a (C) coexpression heatmap. $(A, B)$ Genes belonging to a category are denoted with " $Y$ "; the categories used are SM (specialized metabolism), TPS (terpene synthases, diamond shape vertices) and CYP (cytochrome P450s, square shaped vertices). (A,B) Corresponding expression fold change (FC) increase 24 hours after pathogen inoculation. (D) The hypergeometric test was used to calculate P-values for GO term enrichment based on the GO-basic database and all genes with $M R<100$. The P-values were adjusted using the Bonferroni-Holm method and showed that the top 5 enriched GO terms are associated with biotic stress responses. (E) Summarized diagram of the maize kauralexin biosynthesis pathway showing genes highly coexpressed with the reference gene AN2 in red. 